\title{
TWISTOR CR MANIFOLDS AND THREE-DIMENSIONAL CONFORMAL GEOMETRY
}

BY

CLAUDE R. LEBRUN ${ }^{1}$

\begin{abstract}
A CR (i.e. partially complex) 5-manifold is contructed as a sphere bundle over an arbitrary 3-manifold with conformal metric. This so-called twistor $C R$ manifold is show to capture completely the original geometry, and necessary and sufficient conditions are given for an abstract CR manifold to arise via the construction. The above correspondence is then used to prove that a twistor CR manifold is locally imbeddable as a real hypersurface in $\mathbf{C}^{3}$ only if it is real-analytic with respect to a suitable atlas.
\end{abstract}

Introduction. There is a very beautiful interplay between the conformal geometry of Riemannian manifolds and complex analysis, examples of which are found both in the classical theorem asserting the existence of isothermal coordinates on a surface and in the theory of self-dual Riemannian 4-manifolds (Penrose [1976], Atiyah et al. [1978]). Intermediating between these two theories is a relationship between 3dimensional conformal geometry and CR (partially complex) manifolds of dimension 5. In this paper, we will explain this relationship, focusing on the imbedding problem: When can these partially complex manifolds be realized as real hypersurfaces in complex 3-manifolds?

The idea of associating a CR manifold with a conformal Riemannian 3-manifold originated with Penrose $[\mathbf{1 9 7 5}, \mathbf{1 9 8 3}]$, who, starting with a real-analytic space-like hypersurface in a real-analytic Lorentzian 4-manifold, constructed a real hypersurface in a complex 3-manifold. If the space-like hypersurface is totally geodesic (or merely all-umbilic), Penroses's CR manifold coincides, in our terminology, with the "twistor CR manifold" of the conformal structure induced on the space-like hypersurface.

After establishing notation and conventions in $\S 0$, principal results on $\mathrm{CR}$ manifolds are reviewed and the notions of CR vector bundle and CR contact form are introduced. In $\S 1$, a general machine for constructing involutive distributions is produced, and in $\$ 2$ this construction is used to associate to every conformal Riemannian 3-manifold a CR 5-manifold. The CR manifolds arising in this way are characterized abstractly in $\S 4$ in terms of properties presented in $\S 3$. Then, in $\S 5$, it is shown that these CR manifolds are isomorphic to real hypersurfaces of complex

Received by the editors March 15, 1983 and, in revised form, July 8, 1983.

1980 Mathematics Subject Classification. Primary 32F25; Secondary 53A30, 83C 99.

${ }^{1}$ Work supported in part by NSF grant MCS-812-0790. 
manifolds only in the case that the original conformal structure is analytic with respect to a suitable atlas.

The author would like to acknowledge the many helpful conversations with Roger Penrose which originally stimulated this paper, and to thank the Mathematical Sciences Research Institute for providing an atmosphere conducive to completion of this work.

0. Preliminaries. (a) $C R$ manifolds. Let $X^{n}$ be a smooth manifold, and let $\mathbf{C} T X:=\mathbf{C} \otimes_{\mathbf{R}} T X$ denote its complexified tangent bundle. A distribution of complex $m$-planes $D$ on $X$ will mean a smooth rank $m$ complex bundle $D \rightarrow X$ together with an inclusion $D \hookrightarrow \mathrm{CTX}$. We will say that $D$ is totally complex if $D \cap \bar{D}$ is the zero section $0_{X} \subset \mathbf{C T X}$, where the overbar denotes complex-conjugation in CTX. We will say that $D$ is involutive if $[\mathscr{E}(D), \mathscr{E}(D)] \subset \mathscr{E}(D)$ - that is, if the space of smooth sections of $D$ is closed under Lie brackets. If $D$ is totally complex and involutive, and if $n=2 m+1$, we will say that $(X, D)$ is a $C R(n-)$ manifold and say that $D$ is a $C R$ structure for $X$. Following a standard abuse of terminology, we will say that $X$ is a CR manifold if $D$ is understood.

EXAMPLE. Let $\mathfrak{M}^{N}$ be a complex $N$-manifold, and let $X^{2 N-1} \subset \mathfrak{M}$ be a smooth real hypersurface. Let $T^{\prime} \mathfrak{M} \subset \mathbf{C} T \mathfrak{M}$ denote the holomorphic tangent bundle of $\mathfrak{M}$, the $+i$ eigenspace of the almost complex structure tensor $J$, spanned in local coordinates $\left(z^{1}, \ldots, z^{N}\right)$ by $\left(\partial / \partial z^{1}, \ldots, \partial / \partial z^{N}\right)$. Then $\overline{T^{\prime} \mathfrak{M}}$ is an involutive, totally complex distribution on $\mathfrak{M}$. One easily checks that $D:=\mathrm{C} T X \cap \overline{T^{\prime} \mathfrak{M}}$ has constant rank $N-1$, and it is immediate that this distribution is totally complex and involutive. Thus, $(X, D)$ is a $\mathrm{CR}$ manifold.

$\mathrm{CR}$ manifolds given in this way will be called imbedded $\mathrm{CR}$ manifolds. If, near each $x \in X,(X, D)$ is isomorphic to an embedded CR manifold, we will say that $X$ is locally imbeddable; if a global isomorphism with an imbedded CR manifold exists, then $\mathrm{M}$ will be called imbeddable. For the CR manifolds of interest in this paper, local and global imbeddability will turn out to be equivalent.

Note that any real-analytic CR manifold is imbeddable, as readily follows from either Cauchy-Kowalewski or the Frobenius theorem applied to a complexification.

Let $(X, D)$ be a CR manifold. Then, letting $\mathscr{E}^{p}:=\mathscr{E}\left(\Lambda^{p} \mathbf{C} T^{*} X\right)$ denote the sheaf of smooth $p$-forms, we may define locally free subsheaves of $\mathscr{E}^{0}$ modules

$$
\left.\hat{\mathscr{E}}^{p, q}:=\left\{\omega \in \mathscr{E}^{p+q} \mid v_{0} \wedge v_{1} \wedge \cdots \wedge v_{q}\right\lrcorner \omega=0, \forall v_{0}, \ldots, v_{q} \in D\right\}
$$

for $p, q$ nonnegative integers, and set $\mathscr{E}^{p,-1}:=\{0 \in \mathscr{E}\}$. Then $d\left(\hat{\mathscr{E}}^{p, q}\right) \subset \hat{\mathscr{E}}^{p, q+1}$. If we now define the smooth $(p, q)$-forms $\mathscr{E}^{p, q}$ by

$$
\mathscr{E}^{p, q}:=\hat{\mathscr{E}}^{p, q} / \hat{\mathscr{E}}^{p+1, q-1} \cong \mathscr{E}\left(\Lambda^{p} D^{\perp} \otimes \Lambda^{q} D^{*}\right)
$$


there is a natural family of maps $\bar{\partial}$ defined by the requirement that the diagrams

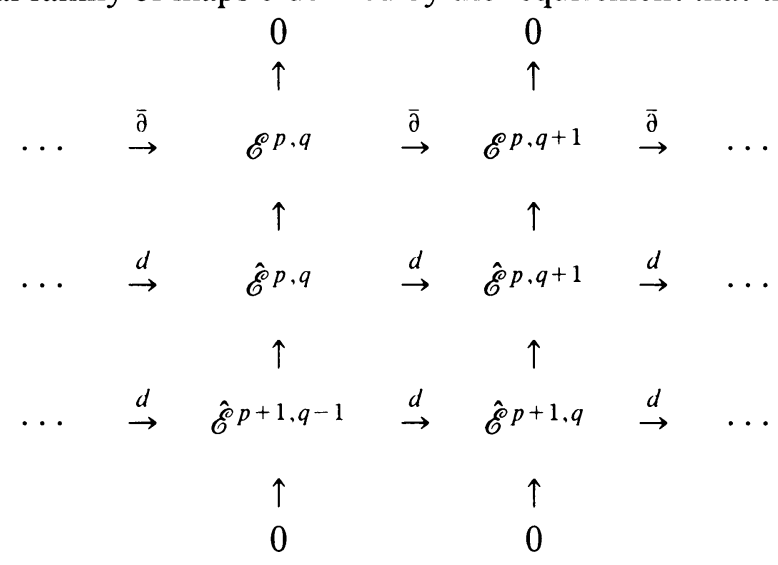

should commute. Setting $\Omega^{p}:=\operatorname{Ker}\left(\bar{\partial}: \mathscr{E}^{p, 0} \rightarrow \mathscr{E}^{p, 1}\right)$, we have constructed a cochain complex

$$
0 \rightarrow \Omega^{p} \rightarrow \mathscr{E}^{p, 0} \stackrel{\bar{\partial}}{\rightarrow} \mathscr{E}^{p, 1} \stackrel{\bar{\partial}}{\rightarrow} \cdots \stackrel{\bar{\partial}}{\rightarrow} \mathscr{E}^{p, m} \rightarrow 0
$$

for each $p$, called the pth $\bar{\partial}$ complex of $(X, D)$. We will often call the 0 th $\bar{\partial}$ complex simply the $\bar{\partial}$ complex of $X$. Elements of $\Omega^{0}$ are called $C R$ functions. Note that $f$ : $X \rightarrow \mathbf{C}$ is a $\mathrm{CR}$ function iff $f_{*} D \subset \operatorname{span}(\partial / \partial \bar{z})$. In the same vein, a smooth mapping $f: X_{1} \rightarrow X_{2}$ between CR manifolds $\left(X_{1}, D_{1}\right)$ and $\left(X_{2}, D_{2}\right)$ will be called a CR (respectively, anti-CR) map if $f_{*} D_{1} \subset D_{2}$ (respectively, $f_{*} D_{1} \subset \overline{D_{2}}$ ).

When $X$ is an imbedded CR manifold, the equation $\bar{\partial} f=0$ defining $\Omega^{0}$ becomes the restriction of the usual Cauchy-Riemann equations to the hypersurface. Hence the terminology "CR (i.e. Cauchy-Riemann) manifold". Note that the operator we denote by $\bar{\partial}$ is called $\bar{\partial}_{X}$ by most authors in order to distinguish it from the analogous Cauchy-Riemann operator of a complex manifold.

The cohomology of the $\bar{\partial}$ complexes of a CR manifold is generally nontrivial even locally (Lewy [1956]). As a consequence, $H^{q}\left(\mathscr{E}^{p \cdot}\right) \neq \check{H}^{q}\left(\Omega^{q}\right)$ in general. It is for this reason that one must exercise particular care in defining $C R$ objects like vector bundles, as we will attempt to do in (c).

(b) The Levi form and the extension problem. Let $(X, D)$ be a CR manifold. Recall that by definition $D+\bar{D}$ has codimension 1 in CTX. Define a complex line-bundle $E \rightarrow X$ by $E:=\mathrm{C} T X / D+\bar{D}$, which inherits a complex conjugation ${ }^{-}: E \rightarrow E$ from CTX. We may now define a Hermitian form

$$
\mathfrak{2}: D \times D \rightarrow E,
$$

called the Levi form of $(X, D)$, by requiring that

$$
\mathfrak{Q}(v, w)=i\langle[v, \bar{w}]\rangle
$$

for any smooth sections $v, w$ of $D$, where the brackets \langle\rangle denote the equivalence class modulo $D+\bar{D}$. Thus if $\phi$ is a real local 1-form orthogonal to $D$ and $\bar{D}$ then, in the local trivialization of $E$ induced by $\phi$,

$$
\mathfrak{Z}(v, w)=i(d \phi)(v, \bar{w})
$$

for $v, w \in D$. 
If $\mathfrak{Z}$ is definite as a quadratic form, we will say that $(X, D)$ is pseudoconvex. If $\mathfrak{Z}$ is nonsingular, inducing an isomorphism $D \rightarrow \bar{D}^{*} \otimes E$, but is not definite, we will say that $(X, D)$ is anticlastic-i.e. bending in two opposite directions. (Unfortunately, there seems to be no standard term for the latter case.) It is with manifolds of the anticlastic type that this paper will concern itself.

Suppose that $X \subset \mathfrak{M}$ is an imbedded $\mathrm{CR}$ manifold. Then every holomorphic function on $\mathfrak{M}$ restricts to a $\mathrm{CR}$ function on $X$, and an examination of power-series representations immediately reveals that the restriction map $\mathcal{O}_{\mathfrak{M}} \rightarrow \Omega_{X}^{0}$ is injective. A Cauchy-integral technique (Bochner [1943], Lewy [1956]) allows one to conclude (Wells [1966]) that if $X$ is anticlastic then this restriction map is surjective not only on the level of germs but also on the level of global sections, provided that $\mathfrak{M}$ is taken to be a suitably small neighborhood of $X$.

It now follows that the local and global imbedding problems for anticlastic CR manifolds are equivalent. Suppose that $X$ is an anticlastic CR manifold covered by open sets $U_{j}$ on which imbeddings $\phi_{j}: U_{j} \rightarrow \mathrm{C}^{N}$ are specified. Then the component functions of

$$
\phi_{j} \circ \phi_{k}^{-1}: \phi_{k}\left(U_{j} \cap U_{k}\right) \rightarrow \mathbf{C}^{N}
$$

are restrictions to $\phi_{k}\left(U_{j} \cap U_{k}\right)$ of holomorphic functions on some neighborhood of $\phi_{k}\left(U_{j} \cap U_{k}\right)$ in $\mathrm{C}^{N}$; therefore $\phi_{j} \circ \phi_{k}^{-1}$ is the restriction of a biholomorphism

$$
\Phi_{j k}: V_{j k} \rightarrow V_{k j} \subset \mathbf{C}^{N} \text {. }
$$

Hence an ambient space $\mathfrak{M}$ may be constructed by gluing together regions in $\mathbf{C}^{N}$ with the transition functions $\Phi_{j k}$.

Also note that, for $X \subset \mathfrak{M}$ an imbedded CR manifold, there is a natural restriction map $\mathcal{O}\left(\Lambda^{p} T^{*} \mathfrak{M}\right) \rightarrow \Omega_{\chi}$. By considering local trivializations of $\Lambda^{p} T^{*} \mathfrak{M}$, one sees that sections of $\Omega_{X}^{p}$ extend to a neighborhood of $X$ as sections of $\Omega_{\mathfrak{R}}^{p}:=\mathcal{O}\left(\Lambda^{p} T^{* * \mathfrak{M})}\right.$ if $X$ is anticlastic. In this respect there is no essential difference between $\mathrm{CR}$ forms and CR functions.

(c) $C R$ vector bundles. Let $(X, D)$ be a CR manifold, and let $V \rightarrow X$ be a complex vector bundle. A linear map $\nabla: \mathscr{E}(V) \rightarrow \mathscr{E}\left(V \otimes D^{*}\right)$ will be called a pre- $\bar{\partial}$ operator if

$$
\nabla(f \sigma)=f \nabla \sigma+\sigma \otimes \bar{\partial} f
$$

for $\sigma \in \mathscr{E}(V), f \in \mathscr{E}$. Thus, $\nabla$ is a sort of "partial connection" on $V$, with covariant derivatives only defined for complex directions in $D$.

For every pre- $\bar{\partial}$ operator $\nabla$ on $V \rightarrow X$, there is a natural family of associated first-order differential operators

$$
\nabla^{p, q}: \mathscr{E}^{p, q}(V) \rightarrow \mathscr{E}^{p, q+1}(V),
$$

where $\mathscr{E}^{p, q}(V):=\mathscr{E}^{p, q} \otimes_{\mathscr{E}} \mathscr{E}(V) \cong \mathscr{E}\left(V \otimes \Lambda^{p} D^{\perp} \otimes \Lambda^{q} D^{*}\right)$; these may be defined by setting $\nabla^{p, q}(\sigma \otimes \phi)=(\nabla \sigma) \wedge \phi+\sigma \otimes \bar{\partial} \phi$ for $\sigma \in \mathscr{E}(V), \phi \in \mathscr{E}^{p, q}$, where $\wedge$ is the product induced by $\wedge: \mathscr{E}^{r}(V) \otimes \mathscr{E}^{s} \rightarrow \mathscr{E}^{r+s}(V)$. We will now define $\nabla$ to be a $\bar{\partial}$ operator if $\nabla^{0,1} \nabla^{0,0}=0$; i.e. if our partial connection is flat. We will often simply use the notation $\bar{\partial}: \mathscr{E}^{p, q}(V) \rightarrow \mathscr{E}^{p, q+1}(V)$ to denote $\nabla^{p, q}$ for an understood fixed $\bar{\partial}$ operator $\nabla$. The pair $(V, \nabla)$ will be called a $C R$ vector bundle. 
With the above definition, the "Leibnitz rule" for $d$ implies $\bar{\partial}^{2}=0$, and we have a family of complexes

$$
0 \rightarrow \Omega^{p}(V) \rightarrow \mathscr{E}^{p, 0}(V) \underset{\bar{\partial}}{\rightarrow} \mathscr{E}^{p, 1}(V) \underset{\bar{\partial}}{\rightarrow} \mathscr{E}^{p, 2}(V) \rightarrow \cdots,
$$

where $\Omega^{p}(V):=\operatorname{Ker}\left[\bar{\partial}^{p, 0}\right]$ is the space of CR $p$-forms with values in $V$.

EXAMPLE 1. Let $V_{p}:=\Lambda^{p} D^{\perp} \subset \Lambda^{p} \mathrm{C} T X$. Then the standard $\bar{\partial}$ operator $\bar{\partial}: \mathscr{E}^{p, 0} \rightarrow$ $\mathscr{E}^{p, 1}$ may be reinterpreted as a $\bar{\partial}$ operator $\nabla: \mathscr{E}\left(V_{p}\right) \rightarrow \mathscr{E}^{0,1}\left(V_{p}\right)$; the fact $\nabla^{0,1} \nabla^{0,0}=0$ is the standard statement that $\bar{\partial}^{2}=0$. Thus $\left(V_{p}, \bar{\partial}\right)$ is a CR vector bundle.

EXAMPLE 2. Let $V$ be a vector bundle on $X$ equipped with a family of local trivializations such that the transition functions are matrix-valued CR functions. Then letting $\bar{\partial}$ act on sections of $V$ by componentwise differentiation makes $V$ a CR vector bundle.

CR bundles of the above type will be called locally trivial.

EXAMPLE 3. Let $X \subset \mathfrak{M}$ be an imbedded CR manifold. Then every holomorphic vector on $\mathfrak{M}$ restricts to $X$ as a locally trivial CR vector bundle.

(d) $C R$ contact structures. If $Y^{2 m+1}$ is a smooth manifold, and if $P \subset T Y$ is a distribution of real $2 m$-planes, one says that $P$ is a contact structure for $Y$ if the Frobenius integrability obstruction

$$
\begin{aligned}
& F: P \times P \mapsto T Y / P \\
& :(v, w) \mapsto\langle[v, w]\rangle
\end{aligned}
$$

is nondegenerate. An equivalent way of stating this is that $P$ is a contact structure iff the tautological line-bundle valued 1-form $\nu: T Y \rightarrow L=T Y / P$ satisfies $\nu \wedge(d \nu)^{\wedge m}$ $\neq 0$, where $d \nu$ may be computed in terms of any local trivilization of $L$. In point of fact, $\left.d \nu\right|_{P}=F$ is a well-defined $L$-valued 2-form on $L$, so $L^{\otimes(m+1)}$ is canonically isomorphic to $\Lambda^{2 m+1} T Y$ via $\nu \wedge(d \nu)^{\wedge m}$. The 1-form $\nu$ (or sometimes its representation in terms of a local trivialization of $L$ ) is called a contact form on $Y$.

EXAMPLE. Let $(X, D)$ be a CR manifold with nondegenerate Levi form. Then $T X \cap(D+\bar{D})$ is a contact structure for $X$. Warning: This is not a "CR contact structure" in the sense we will introduce presently.

In analogy to the above a holomorphic contact structure on a complex $(2 m+1)$ manifold $\mathfrak{M}$ is defined to be a holomorphic distribution of $2 m$-planes $P \subset T^{\prime} \mathfrak{M}$ with nondegenerate Frobenius integrability obstruction

$$
\begin{gathered}
F: P \times P \rightarrow T^{\prime} \mathfrak{M} / P \\
:(v, w) \rightarrow\langle[v, w]\rangle .
\end{gathered}
$$

Equivalently, a holomorphic contact structure is a holomorphic line-bundle valued 1 -form $\Theta: T^{\prime} \mathfrak{M} \rightarrow \mathbf{L}$ with $\Theta \wedge(\partial \Theta)^{\wedge m} \neq 0$. Clearly $\mathbf{L}^{\otimes(m+1)} \cong \Lambda^{2 m+1} T^{*} \mathfrak{M}$.

Now if $X \subset \mathfrak{M}$ is a smooth hypersurface, we may consider the restriction of a holomorphic contact structure to $X$. An abstraction from the resulting object results in the following

Definition. Let $(X, D)$ be a CR $(4 m+1)$-manifold and let $\mathbf{L} \rightarrow X$ be a $C R$ line-bundle. Then a 1-form

$$
\theta \in \Omega^{1}(\mathbf{L}):=\operatorname{Ker}\left[\bar{\partial}: \mathscr{E}^{1,0}(\mathbf{L}) \rightarrow \mathscr{E}^{1,1}(\mathbf{L})\right]
$$


will be called a $C R$ contact form if $\theta \wedge(d \theta)^{\wedge m} \neq 0$. If $\theta \in \Omega^{1}(\mathbf{L})$ and $\hat{\theta} \in \Omega^{1}(\hat{\mathbf{L}})$ are two such forms, we will say they are equivalent if there exists an isomorphism $\Psi$ : $\mathbf{L} \rightarrow \hat{\mathbf{L}}$ with

$$
\hat{\theta}=\Psi \circ \theta: \mathbf{C} T X \rightarrow \hat{\mathbf{L}} .
$$

An equivalence class of CR contact forms will be called a $C R$ contact structure for $X$.

Clearly $\mathbf{L}^{\otimes m+1} \cong \Lambda^{2 m+1}\left(T^{\prime} X\right)$ as CR line-bundles, where $T^{\prime} X:=\mathbf{C} T X / D$. As in the smooth and holomorphic cases, there is a one-one correspondence between such structures and appropriate distributions.

1. Hamiltonian distributions and CR structures. Let $M$ be a smooth $n$-manifold, and let $\mathscr{X}:=\mathbf{C} T^{*} M$ denote the complexified cotangent bundle of $M$; let $\pi: \mathscr{X} \rightarrow M$ denote the canonical projection. Let $A \subset \mathbf{C T} \mathscr{X}$ denote the antiholomorphic tangent bundle of the fibers of $\pi$. Thus, if $\left(\tilde{x}^{j}, j=1, \ldots, n\right): U \rightarrow \mathbf{R}^{n}$ is a coordinate system for $U \subset M$, and if $x^{j}, \xi_{j}, \eta_{j}, \zeta_{j}$ are the functions on $\pi^{-1}[U]$ given by

$$
\begin{gathered}
x^{j}:=\pi^{*} \tilde{x}^{j}: x \rightarrow \tilde{x}^{j}(\pi(x)), \\
\left.\zeta_{j}:=\right\lrcorner_{\partial / \partial \tilde{x}^{j}}: x \rightarrow\left\langle x, \partial / \partial \tilde{x}^{j}\right\rangle, \\
\xi_{j}:=\operatorname{Re} \zeta_{j}, \quad \eta_{j}:=\operatorname{Im} \zeta_{j},
\end{gathered}
$$

then $\left.A\right|_{\pi^{-1}[U]}$ is spanned in the coordinates $\left(\left(x^{j}\right),\left(\xi_{j}\right),\left(\eta_{j}\right)\right)[j=1, \ldots, n]$ by $\left\{\partial / \partial \bar{\zeta}_{j}\right\}$.

If $\mathscr{X} \rightarrow M$ were given as an abstract complex vector bundle, some added piece of information would be necessary to identify $\mathscr{X}$ with $\mathrm{C} T^{*} M$. This extra information is supplied by the canonical 1 -form $\theta$ of $\mathscr{X}$, given by the formula

$$
\left.\langle\theta, w\rangle\right|_{x \in \mathscr{X}}:=\left\langle x, \pi_{* x} w\right\rangle,
$$

where $w$ is any vector field on $\mathscr{X}$. In the former local coordinates for $\pi^{-1}[U]$, we have $\theta=\sum_{j=1}^{n} \zeta_{j} d x^{j}$. We will be particularly concerned here with the 2 -form $\omega:=d \theta$, which we will call the Hamiltonian form of $\mathscr{X}$; thus, $\omega$, which is given by $\omega=$ $\sum_{j=1}^{n} d \zeta_{j} \wedge d x^{j}$ in our local coordinates, is the complexified analog of the usual symplectic form of $T^{*} M$ (cf. Weinstein [1977]).

Let $T^{\prime} \mathscr{X}:=\mathbf{C} T \mathscr{X} / A$, and let $T^{*} \mathscr{X}$ denote its dual $A^{\perp} \subset \mathbf{C} T^{*} \mathscr{X}$. Then the contraction map

$$
\lrcorner_{\omega}: \mathbf{C T X} \rightarrow \mathbf{C} T^{*} \mathscr{X}: w \rightarrow \omega(w, \cdot)
$$

has image in $T^{\prime *} \mathscr{X}$ and has $A$ for its kernel. Thus, the induced map $\lrcorner_{\omega}: T^{\prime} \mathscr{X} \rightarrow T^{*} \mathscr{X}$ is an isomorphism. This fact will be central to the discussion.

Now consider a codimension 2 submanifold $\mathscr{Y} \subset \mathscr{X}$. If, for every $p \in M$, the real variety $\mathscr{Y} \cap \pi^{-1}(p)$ is a complex hypersurface in the complex vector space $\pi^{-1}(p)$, we will say that $\mathscr{Y}$ is an energy surface. If, in addition, the conormal bundle $\left.N_{p}^{*} \subset T^{*}\left(\pi^{-1}(p)\right)\right|_{\mathscr{Y} \cap \pi^{-1}(p)}$ of $\mathscr{Y} \cap \pi^{-1}(p)$ relative to $\pi^{-1}(p)$ contains no nonzero covector corresponding to a real vector under the natural double-dual identification of $T^{*}\left(\pi^{-1}(p)\right)$ with $\left.\pi^{*} \mathbf{C} T M\right|_{\pi^{-1}(p)}$ for every $p \in M$, we will say that $\mathscr{Y}$ is generic.

Let $\tilde{\omega}$ denote the restriction of $\omega$ to an energy surface $\mathscr{Y}$, and let $D$ denote the kernel of $\lrcorner_{\tilde{\omega}}: \mathbf{C T Y} \rightarrow \mathbf{C} T^{*} \mathscr{Y}$. 
Proposition. $D$ is an involutive distribution of complex $n$-planes on $\mathscr{Y}$.

If $\mathscr{Y}$ is generic, $\mathrm{D}$ contains no nonzero real vectors.

COROLlaRY. If $M$ is a 3-manifold and $\mathscr{Y} \subset \mathbf{C} T^{*} M$ is a generic energy surface, then $D$ is a $C R$ structure for $\mathscr{Y}$.

Definition. The distribution $D$ of the proposition will be called the Hamiltonian distribution of $\mathscr{Y}$.

Proof of the Proposition. Let us begin by checking that $D$ has constant dimension $n$. To this end, let $A \subset \mathrm{C} \mathscr{X}$ denote, as before, the antiholomorphic tangent bundle of the fibers of $\mathscr{X} \rightarrow M$, and introduce $B:=A \cap \mathbf{C} T \mathscr{Y}$, which is a distribution of complex $(n-1)$-planes on $\mathscr{Y}$. Defining $T^{\prime} \mathscr{Y}:=\mathrm{C} T \mathscr{Y} / B$, we need to show that the kernel $C$ of

$$
\hat{\lrcorner}_{\tilde{\omega}}: T^{\prime} \mathscr{Y} \rightarrow T^{*} * \mathscr{Y}:=B^{\perp}
$$

has constant dimension 1. But $\tilde{\omega}$ is a skew form on the odd-dimensional complex bundle $T^{\prime} \mathscr{Y}$, and so $C$ must be nontrivial at every point of $\mathscr{Y}$.

Since $\hat{\lrcorner}_{\omega}$ is an isomorphism, the dimension of the kernel $C$ of $\hat{\lrcorner}_{\tilde{\omega}}$ cannot exceed the codimension of $\left.T^{\prime} \mathscr{Y} \subset\left(T^{\prime} \mathscr{X}\right)\right|_{\mathscr{y}}$, which is one by hypothesis. Hence $C$ has constant dimension 1 and $D$ has constant dimension $n$.

Secondly, we must show that $D$ is involutive-which will follow rather easily from the fact that $\tilde{\omega}$ is a closed form. Indeed, if $v$ and $w$ are complex vector fields on $\mathscr{Y}$ satisfying $v\lrcorner \tilde{\omega}=w\lrcorner \tilde{\omega}=0$, then

$$
\left.\left.\left.\left.\left.[v, w]\lrcorner \tilde{\omega}=L_{v}(w\lrcorner \tilde{\omega}\right)-w\right\lrcorner L_{v} \tilde{\omega}=-w\right\lrcorner[v\lrcorner d \tilde{\omega}+d(v\lrcorner \tilde{\omega}\right)\right]=0 .
$$

Lastly, we must verify that if $\mathscr{Y}$ is generic then $D$ contains no nonzero real vectors. Letting $j: \bar{A} \hookrightarrow T^{\prime} \mathscr{X}$ be the canonical inclusion and letting $\tau: \pi^{*} \mathbf{C} T M \cong \bar{A}^{*}$ be the tautological double-duality isomorphism, one readily checks that

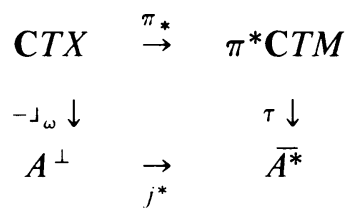

is commutative by inspecting the appearance of $\omega$ in the previously used local coordinates. If $D$ contains a real vector $v \neq 0, \tau \pi_{*}(v)$ is real (in the sense featuring in the definition of a generic energy surface). Moreover, $v \notin A$, so $v$ is transverse to the fibers of $\pi$ and hence $\tau \pi_{*}(v) \neq 0$. But since $v \in D, \omega(v, \cdot)$ is normal to $\mathscr{Y}$, and hence $\left.-j^{*}\right\lrcorner_{\omega}(v)=\tau \pi_{*} v$ is an element of the holomorphic cornormal bundle of the relevant fiber of $\mathscr{Y} \rightarrow M$. This implies that $\mathscr{Y}$ is not generic, so the claim follows by contraposition.

2. The twistor CR manifold of a 3-manifold. Now let $M$ denote a smooth 3-manifold equipped with a smooth Riemannian conformal structure-i.e. endowed with a class $[g$ ] of Riemannian metrics of the form

$$
[g]=\{f g \mid f \in \mathscr{E}(M), f>0\},
$$


where $g$ is some fixed smooth Riemannian metric on $M$. Associated to every Riemannian metric $g$ there is a "cometric" $g^{-1}$, which may be considered as a positive definite quadratic form on $T^{*} M$, defined by $g^{-1}(g(v, \cdot), \cdot)=v, \forall v \in T M$. By bilinear extension, $g^{-1}$ becomes a complex quadratic form on $C T^{*} M$, and we may define a 7-manifold

$$
\hat{N}:=\left\{\tau \in \mathbf{C} T^{*} M \mid g^{-1}(\tau, \tau)=0, \tau \neq 0\right\},
$$

as well as the 5-manifold $N$ which is the projective image of $\hat{N}: N:=\hat{N} / \mathbf{C}_{*}$, where the nonzero complex numbers $\mathbf{C}_{*}$ act freely on $\hat{N}$ by scalar multiplication. Clearly both $N$ and $\hat{N}$ depend only on [ $g$ ], and not $g$ itself; moreover, $[g]$ is determined by either. (We may characterize $N$ abstractly as a nonsingular conic subbundle of $\mathbf{P}\left(\mathbf{C} T^{*} M\right)$ which is complex-conjugation invariant and contains no real points; every such subbundle arises from a unique $[g]$.)

I claim that $\hat{N} \subset \mathrm{C} T^{*} M$ is generic in the sense of $\S 1$; thus, by the Corollary to the Proposition, the Hamiltonian distribution of $\hat{N}$ is a CR structure. We also see that this distribution projects to give $N$ a CR structure; this CR 5-manifold wll be called the twistor manifold of $(M,[g])$ as an indication of the relationship of this object to the work of Penrose.

To see that $\hat{N}$ is generic, we may identify $T_{m}^{*} M, m \in M$, with $\mathbf{R}^{3}$ by choosing an orthonormal basis, thus inducing an identification of $\hat{N} \cap \mathrm{C} T_{m}^{*} M$ with

$$
\left\{\left(z_{1}, z_{2}, z_{3},\right) \in \mathbf{C}^{3}-\{0\} \mid \sum_{j=1}^{3} z_{j}^{2}=0\right\} .
$$

Under this identification, the conormal bundle of the fiber of $\hat{N}$ over $m$ is spanned at $\left(z_{1}, z_{2}, z_{3}\right)$ by the real and complex parts of $\sum_{j=1}^{3} z_{j} d z_{j}$. For this conormal space to contain a "real" covector (that is, a covector normal to $i \mathbf{R}^{3} \subset \mathbf{C}^{3}$ ) would require the existence of $\alpha \in \mathbf{C}_{*}$ with

$$
\left.0=\left[\sum_{j}\left(\alpha z_{j} d z_{j}+\bar{\alpha} \bar{z}_{j} d \bar{z}_{j}\right)\right]\right\lrcorner \partial / \partial y^{k}=i\left(\alpha z_{k}-\bar{\alpha} \bar{z}_{k}\right), \quad k=1,2,3 .
$$

(We have followed standard conventions in coordinatizing $\mathbf{C}^{3}$ by $\left\{x_{j}:=\operatorname{Re} z_{j}\right\} \cup$ $\left\{y_{j}:=\operatorname{Im} z_{j}\right\}$.) Hence $\left(\alpha z_{j}\right) \neq 0$ would have real components and satisfy $\sum_{j=1}^{3}\left(\alpha z_{j}\right)^{2}$ $=0$, yielding a contradiction. So $\hat{N}$ is indeed generic, and its Hamiltonian distribution is a CR structure.

We now consider the projectification $\mathbf{P}: \hat{N} \rightarrow N:=\hat{N} / \mathbf{C}_{*}$. Since the scalar multiplication map

$$
m_{\alpha}: \hat{N} \rightarrow \hat{N}: y \rightarrow \alpha y, \quad \alpha \in \mathbf{C}_{*},
$$

has the property that $m_{\alpha}^{*} \theta=\alpha \theta$ and hence (taking exterior derivatives) $m_{\alpha}^{*} \tilde{\omega}=\alpha \tilde{\omega}$, it follows that $\left.D:=\operatorname{ker}(\lrcorner_{\tilde{\omega}}\right)$ is invariant under the action of $\mathbf{C}_{*}$. The fibers of $\mathbf{P}$ are contained in the fibers of $\pi$, where $D$ coincides with $B:=A \cap \mathbf{C} T \hat{N}$; the fibers of $\mathbf{P}$ just being the straight complex lines through 0 in the fibers of $\mathscr{X} \rightarrow M$, we see that $D \cap \mathbf{C} T\left[\mathbf{P}^{-1}(a)\right]$ is 1-dimensional for every $a \in N$. Hence $\not:=\mathbf{P}_{*} D$ is a distribution of complex 2-planes on $N$. As $w \in D$ can have its imaginary part tangent to 
$\mathbf{P}^{-1}(a)$ only if its real part is also tangent, $Д$ contains no real vectors. Also, notice that the distribution $\not$ is involutive because $D$ is. Therefore $\square$ is a CR structure.

Thus, in summary, we have associated to every smooth 3-manifold with conformal structure $(M,[g])$ a CR 5-manifold $N$ by means of the Hamiltonian form of $\mathbf{C} T^{*} M$. In what follows, we will first characterize in abstracto all CR manifolds that arise via this process, and then answer the question: when can $N$ be realized as a hypersurface in a complex manifold? The answer to the latter question will turn out to be that $N$ can be so realized iff $(M,[g])$ can be given a real-analytic structure. Note that if $(M,[g])$ is real-analytic, so is $(N, I)$; thus nonimbeddability appears in the largest number of cases conceivable!

The reader may note that we have already narrowed our scope more than might be considered strictly necessary; for in the above construction of $N$ we might have, for instance, chosen to consider submanifolds of $\mathbf{P}\left(\mathbf{C} T^{*} M\right)$ defined by homogeneous polynomials that are not quadratic, or have chosen to also consider the case when $M$ has a higher dimension simply by choosing a more general definition of a CR manifold. We believe, however, that the main results of this article will justify these restrictions; generalizations will be left to the reader's imagination.

3. The structure of twistor CR manifolds. So far, we have constructed a CR 5-manifold $(N, Д)$ for every 3-manifold $M$ with conformal metric [ $g$ ]; the 5-manifold $N$ in the construction is just the space of null directions in the complex cotangent bundle $\mathbf{C} T^{*} M$. In this section, we identify several important properties of $(N, Д)$ which, as we will see later, suffice to characterize $C R$ manifolds arising via the construction.

Before going any further, however, it is best to point out that the manifold $N$ is familiar to the reader in another guise. Suppose, for simplicity, that $M$ is oriented and that a particular metric $g$ has been chosen to represent the fixed conformal class. Then we may identify $N \rightarrow M$ with the sphere bundle $S M \rightarrow M$, given by

$$
S M:=\{v \in T M \mid g(v, v)=1\} .
$$

To see this, let $e \in \mathscr{E}\left(A^{3} T M\right)$ be the positive unit 3-form, so that $e(x)=e_{1} \wedge e_{2} \wedge e_{3}$ for $\left(e_{1}, e_{2}, e_{3}\right)$ an oriented basis for $T_{x} M, x \in M$. Then $e$ may be used to identify $T M$ with $\Lambda^{2} T^{*} M$ by contraction. But $N$ maps diffeomorphically onto the unit sphere-bundle of $\Lambda^{2} T^{*} M$ via

$$
\phi \mapsto \frac{i \phi \wedge \bar{\phi}}{\|i \phi \wedge \bar{\phi}\|}=\frac{\operatorname{Re} \phi \wedge \operatorname{Im} \phi}{\|\operatorname{Re} \phi \wedge \operatorname{Im} \phi\|} .
$$

Moreover, this map is conformal on the fibers of $N \rightarrow M$, since $\mathrm{SO}(3)$ acts simultaneously as a family of holomorphic maps on the fibers of $N$ and as a family of isometries on the fibers of $S M$ acting transitively on the unit vectors in $T S^{2}$.

Notice that in the nonorientable case we must replace $S M$ with the unit spherebundle of $\Lambda^{2} T^{*} M \cong T M \otimes \Lambda^{3} T^{*} M$.

We now list some properties of $(N, Д)$.

(a) $N$ is foliated by Riemann spheres. The fibers of $\pi: N \rightarrow M$ are all projective complex lines $\mathbf{P}_{1}$, since by construction these fibers are nondegenerate conics whose antiholomorphic tangent spaces are contained in $I$. 
(b) The Levi form of $(N$, Д) is nondegenerate. In fact, the orthogonal space of $\bar{L}+\overline{\bar{L}}$ in $\mathbf{C} T^{*} N$ is spanned by the standard contact form $\sum_{j=1}^{3} p_{j} d x^{j}$ on the sphere bundle $S M$ under the above correspondence, since

$$
b\left[\pi_{*[\phi]} Д\right]=\operatorname{span}(\phi)
$$

and ker $\pi_{*} \subset Д+\bar{д}$. (Here b: $\mathbf{C T M} \rightarrow \mathbf{C} T^{*} M: v \mapsto g(v, \cdot)$.)

With (a) this implies that $(N, Д)$ is anticlastic. For the only alternative would be for $N$ to be pseudoconvex. Yet if $v$ is a holomorphic vector field on one of the $\mathbf{P}_{1}$ 's foliating $N$ we have $[v, \bar{v}]=0$, and hence $\mathfrak{Z}(\bar{v}, \bar{v})=0$, so $\mathfrak{Q}$ is not a definite form.

(c) $(N$, L) has a CR contact structure orthogonal to the foliation (a). The 1-form $\boldsymbol{\theta}=\sum_{j=1}^{3} \zeta_{j} d z^{j}$ on $\mathbf{C} T^{*} M$, when restricted to $\hat{N} \subset \mathbf{C} T^{*} M$, is an element of $\mathscr{E}^{1,0}=\hat{\mathscr{E}}^{1,0}$, since $v \in D_{\phi} \Rightarrow b\left(\pi_{*} v\right)=\alpha \phi$ for some $\alpha \in \mathbf{C}$, so that

$$
\left.\langle\theta, v\rangle\right|_{\phi}=\left\langle\phi, \pi_{*} v\right\rangle=g^{-1}(\phi, \alpha \phi)=0
$$

because $\phi \in \hat{N} \Rightarrow g^{-1}(\phi, \phi)=0$. Moreover, $\bar{\partial} \theta=0$ since $\left.\left.D:=\operatorname{Ker}(\lrcorner_{\tilde{\omega}}=\right\lrcorner_{d \theta}\right)$. So $\theta \in \Omega^{1}$. But, letting $m_{\alpha}: \hat{N} \rightarrow \hat{N}$ be scalar multiplication, $m_{\alpha}^{*} \theta=\alpha \theta$. Let $L \rightarrow N$ be the unique CR line-bundle such that $\hat{N} \rightarrow N$ is the frame bundle for $L^{*}$. Thus $\hat{N}$, equipped with the new $\mathbf{C}_{*}$-action $\alpha \mapsto m_{1 / \alpha}$, is $L-O_{N}$, the frame bundle of $L$. Then there is a unique 1 -form $\theta \in \Omega^{1}(L)$ with $\mathbf{P}^{*} \theta=\theta$. This 1 -form is orthogonal to the fibers of $N \rightarrow M$, since $\theta$ is orthogonal to the fibers of $\hat{N} \rightarrow M$. Moreover, $\theta \wedge d \theta \neq$ 0 , since $\theta \wedge d \theta \neq 0$. Hence $\theta$ is a CR contact form on $N$ of the desired type.

(d) $N$ had an anti-CR involution $\rho: N \rightarrow N$, where by involution we mean $\rho^{2}=1_{N}$, and by anti-CR we mean $\rho_{*} I=\bar{L}$. This map is just the complex conjugation $\mathrm{C} T^{*} M \rightarrow \mathrm{C} T^{*} M$, restricted to $\hat{N}$ and projectivized; this is well defined because $g^{-1}(\bar{\phi}, \bar{\phi})=\overline{g^{-1}(\phi, \phi)}$ and anti-CR because complex conjugation on $\mathbf{C} T^{*} M$ takes $\omega$ to $\bar{\omega}$. Notice that $\rho$ acts without fixed points.

In the $S M$ picture, $\rho$ becomes the antipodal map on the fibers of $S M \rightarrow M$.

(e) The involution $\rho$ respects the foliation (a) and $C R$ contact structure (c) in the sense that $\rho$ maps every leaf of the foliation into itself, and

$$
\rho_{*}\left[\theta^{\perp}\right]=\overline{\theta^{\perp}}
$$

where $\theta^{\perp} \subset \mathrm{C} T N$ is the kernel of $\theta: \mathrm{CTN} \rightarrow L$. The latter assertion follows from the fact that complex conjugation $\mathrm{C} T^{*} M \rightarrow \mathrm{C} T^{*}$ takes $\theta$ to $\bar{\theta}$.

\section{The characterization theorem.}

THEOREM . Let $(\tilde{N}, \tilde{Z})$ be a smooth CR 5-manifold with nondegenerate Levi form and a smooth foliation by $\mathbf{P}_{1}$ 's. Then the space $M$ of leaves of this foliation is a smooth 3-manifold. This manifold admits a Riemannian conformal metric $[g]$ such that $(\tilde{N}, \tilde{L})$ is isomorphic to the twistor $C R$ manifold of $(M,[g])$ iff there exists a CR contact form on $\tilde{N}$ orthogonal to the foliation and respected by an anti-CR involution preserving the foliation.

Proof. Because the leaves of the given foliation are compact, the space $M$ of leaves is Hausdorff. If $\tilde{\pi}: \tilde{N} \rightarrow M$ denotes the canonical projection, and if $x \in M$, then a sufficiently small neighborhood $U(x)$ has as its inverse image $\tilde{\pi}^{-1}[U]$ a 
disc-bundle over the leaf $\tilde{\pi}^{-1}(x)$, since the leaf is compact and some neighborhood of the leaf admits a foliation transverse to the given one. The given foliation defines a flat connection on this disc-bundle; the fact that the leaf $\tilde{\pi}^{-1}(x)$ is simply connected then implies that the holonomy of this connection is trivial. Each fiber of the disc-bundle is therefore mapped homeomorphically onto the neighborhood $U(x)$. The collection of all such mappings then defines an atlas, making $M$ a smooth manifold and $\tilde{\pi}: \tilde{N} \rightarrow M$ a smooth $\mathbf{P}_{1}$-bundle.

If $\tilde{\pi}: N \rightarrow M$ is isomorphic to the twistor CR manifold of $(M,[g])$ for some $[g], \tilde{N}$ admits the required extra structures by $\S 3$. We now suppose these extra structures exist and prove that, conversely, $\tilde{N}$ is the twistor CR manifold of a suitable conformal metric on $M$.

Let $\tilde{\theta} \in \Omega_{N}^{1}(\tilde{L})$ denote the CR contact form of $(\tilde{N}, \tilde{Z})$, where $\lambda: \tilde{L} \rightarrow \tilde{N}$ is some CR line-bundle. Further, let $\hat{\lambda}:\left(\tilde{L}^{*}-O_{\tilde{N}}\right) \rightarrow \tilde{N}$ denote the dual principal $\mathbf{C}_{*}$-bundle, $\tilde{\theta}=\hat{\lambda}^{*} \tilde{\theta}$ the C-valued CR 1-form corresponding to $\tilde{\theta}$, and

$$
\hat{\pi}:=\tilde{\pi} \hat{\lambda}:\left(\tilde{L}^{*}-O_{\tilde{N}}\right) \rightarrow \tilde{M}
$$

the canonical projection. By hypothesis, $\langle\tilde{\theta}, v\rangle=0$ for all vectors $v$ satisfying $\tilde{\pi}_{*} v=0$. Hence for all $y \in\left(\tilde{L}^{*}-O_{\tilde{N}}\right)$ we have $\hat{\theta}_{y}=\hat{\pi}^{*}[\phi(y)]$ for a unique $\phi(y) \in$ $\mathrm{CT}_{x}^{*} M, x:=\hat{\pi}(y)$. This defines a smooth map

$$
\begin{aligned}
& \hat{\Phi}:\left(\tilde{L}^{*}-O_{\tilde{N}}\right) \rightarrow \mathbf{C} T_{x}^{*} M \\
& : y \mapsto \phi(y),
\end{aligned}
$$

which we may projectivize to yield

$$
\begin{gathered}
\boldsymbol{\Phi}: \tilde{N} \rightarrow \mathbf{P}\left(\mathbf{C} T^{*} M\right) \\
: \lambda(y) \mapsto[\phi(y)]
\end{gathered}
$$

since $\hat{\Phi}$ is homogeneous of degree 1 . By construction, $\hat{\Phi}^{*} \theta=\tilde{\theta}$, where $\theta$ denotes the canonical 1-form of $\mathrm{C} T^{*} M$, as in $\S 1$.

We will now examine the restriction of $\Phi$ to a fiber of $\tilde{\pi}$. To this end, let $v$ be a holomorphic vector field on $\tilde{\pi}^{-1}(x)$, and choose a local holomorphic trivialization of the holomorphic line-bundle $\left.\tilde{L}\right|_{\tilde{\pi}^{-1}(x)}$, so that $\tilde{\theta}$ becomes an element of $\mathbf{C} T_{x}^{*} M$ depending on $y \in \tilde{\pi}^{-1}(x)$. Then this dependence is in fact holomorphic, since $\bar{\partial} \tilde{\theta}=0$. But while $\bar{v}\lrcorner d \tilde{\theta}=0$, we also have $v\lrcorner \tilde{\theta} \neq 0$, since $\tilde{\theta} \wedge d \tilde{\theta} \neq 0$ while $v \notin \tilde{\beth}$ and $v\lrcorner \tilde{\theta}=0$. Hence $\left.\Phi\right|_{\tilde{\pi}^{-1}(x)}$ is a holomorphic immersion of $\tilde{\pi}^{-1}(x) \cong \mathbf{P}_{1}$ in $\mathbf{P}\left(\mathbf{C} T^{*} M\right) \cong$ $\mathbf{P}_{2}$.

For clarity, set $\mathbf{P}_{x} \cong \tilde{\pi}^{-1}(x)$ and $K:=\left(\left.\tilde{\not}\right|_{\mathbf{P}_{x}}\right) / \overline{T^{\prime} \mathbf{P}_{x}}$. As a smooth vector-bundle, we therefore have $\left.\tilde{\Delta}\right|_{\mathbf{P}_{x}}=\overline{T^{\prime} \mathbf{P}_{x}}+K$. Since $\mathfrak{\imath}(\bar{v}, \bar{v})=0$, the Levi form

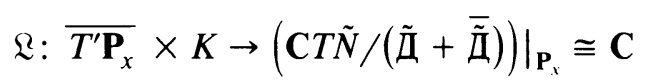

induces an isomorphism $K \cong T^{*} \mathbf{P}_{x}$. Letting $T^{\prime} \tilde{N}:=\mathbf{C} T \tilde{N} / \tilde{\Delta}$, we have

$$
c_{1}\left(\left.T^{\prime} \tilde{N}\right|_{\mathbf{P}_{x}}\right)=c_{1}\left(\left.\mathbf{C} T \tilde{N}\right|_{\mathbf{P}_{x}}\right)-c_{1}\left(\left.\tilde{\boldsymbol{L}}\right|_{\mathbf{P}_{x}}\right)=-2 c_{1} \overline{\left(T^{\prime} \mathbf{P}_{x}\right)}=4
$$


(The odd-degree Chern classes of the complexification of any bundle must be elements of order 2!) But $\theta \wedge d \theta$ is an isomorphism between $\Lambda^{3} T^{\prime} \tilde{N}$ and $\tilde{L}^{\otimes 2}$. Hence

$$
c_{1}\left(\left.\tilde{L}\right|_{\mathbf{P}_{\mathrm{x}}}\right)=\frac{1}{2} c_{1}\left(\left.\Lambda^{3} T^{\prime} \tilde{N}\right|_{\mathbf{P}_{\mathrm{x}}}\right)=\frac{1}{2} c_{1}\left(\left.T^{\prime} \tilde{N}\right|_{\mathbf{P}_{\mathrm{x}}}\right)=2 .
$$

From this we may deduce that the curve $\Phi\left[\mathbf{P}_{x}\right] \subset \mathbf{P}\left(\mathbf{C} T_{x}^{*} M\right)$ is a nondegenerate conic. For since $\left.\Phi\right|_{\mathbf{P}_{x}}$ is an immersion, no projective line can contain $\Phi\left[\mathbf{P}_{x}\right]$; there are no degree 2 immersions $S^{2} \rightarrow S^{2}$ ! But $\left.\Phi\right|_{P_{1}}$ is therefore an imbedding, since if $f$ is a holomorphic function on $\mathbf{P}\left(\mathbf{C} T_{x}^{*} M\right)$ homogeneous of degree 1 -i.e. if $f \in$ $\mathbf{C} T_{x} M$-and if the zero locus of $f$ is the tangent line of $\Phi[\mathbf{P} x]$ at $\Phi(y)$, then

$$
\Phi^{*} f \in H^{0}\left(\mathbf{P}_{x}, \mathcal{O}(2)\right)
$$

has a double zero at $y$ and hence is nonzero elsewhere. ( $\Phi^{*} f$ cannot vanish identically since $\Phi\left[\mathbf{P}_{x}\right]$ cannot lie entirely in the projective line $f=0$.) $\Phi\left[\mathbf{P}_{x}\right]$ is therefore defined by a nonsingular quadratic form $g_{x}^{-1}$, determined up to overall scale by $(\tilde{N}, \tilde{L})$.

The added requirement that $\tilde{\theta}$ is respected by an anti-CR involution $\rho: \tilde{N} \rightarrow \tilde{N}$ guarantees that $g_{x}^{-1}$ may be chosen to the pseudo-Riemannian; for $\Phi(\rho(y))=\overline{\Phi(y)}$ follows from $\rho^{*} \tilde{\theta}=\tilde{\tilde{\theta}}$. Since $\operatorname{dim} M=3$ is odd, so is the signature of $g_{x}^{-1}$; we may therefore take the signature of $g_{x}^{-1}$ to be positive for all $x$. Since $\Phi[\tilde{N}]$ is a smooth manifold, $g_{x}^{-1}$ may be chosen to vary continuous in $x$ over small neighborhoods $U_{\alpha}$ covering $M$; setting $g^{-1}:=\sum_{\alpha} \psi_{\alpha} g^{-1} \alpha$, where $g_{x}^{-1}$ is a positive signature choice for $g_{x}^{-1}$ on $U_{\alpha}$ and where $\psi_{\alpha}$ is a partition of unity subordinate to $\left\{U_{\alpha}\right\}$, then produces a pseudo-Riemannian cometric on $M$ for which

$$
\Phi(\tilde{N})=N=\left\{[\phi] \in \mathbf{P}\left(\mathbf{C} T^{*} M\right) \mid g^{-1}(\phi, \phi)=0\right\} .
$$

To finish the proof, we need merely notice that $\left(\Phi^{-1}\right)^{*} \tilde{\theta}=\theta$, the contact form of $N$, and that the kernel of

$$
\lrcorner_{\theta \wedge d \theta}: \mathbf{C} T N \rightarrow\left(\Lambda^{2} \mathbf{C} T^{*} N\right) \otimes L^{\otimes 2}
$$

is the Hamiltonian distribution $Д$ of $N$. But the kernel of

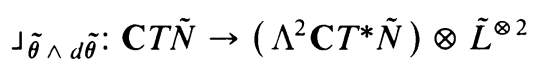

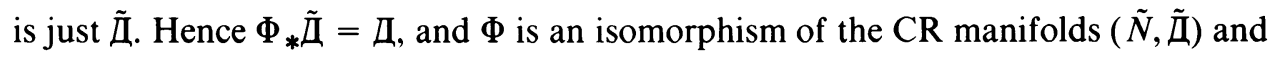
$(N, Д)$. But we finally may conclude that the constructed metric $g$ is positive-definite, for otherwise $Д$ would contain nonzero real vectors.

\section{The nonimbeddabilty theorem.}

Lemma. Let $(N, Д)$ be the twistor $C R$ manifold of some smooth conformal Riemannian 3-manifold $(M,[g])$ and let $\pi: N \rightarrow M$ be the canonical projection. If $\mathbf{P}_{x}:=\pi^{-1}(x), x \in M$, and $\nu \rightarrow \mathbf{P}_{x}$ is the holomorphic (normal) vector bundle given by

$$
\nu:=\left(\left.\mathbf{C T N}\right|_{\mathbf{P}_{\mathbf{v}}}\right) /\left(T^{\prime} \mathbf{P}_{x}+\left.\not\right|_{\mathbf{P}_{\mathbf{v}}}\right)
$$

then $\nu \cong \mathscr{H} \oplus \mathscr{H}$ as a holomorphic bundle, where $\mathscr{H}$ is the hyperplane line-bundle $\left(c_{1}(\mathscr{H})=1\right)$ on $\mathbf{P}_{x} \cong \mathbf{P}_{1}$. 
Proof. In $\S 4$ we saw that $c_{1}\left(\left.T^{\prime} N\right|_{\mathbf{P}_{x}}\right)=4$. Hence

$$
c_{1}(\nu)=c_{1}\left[\left(\left.T^{\prime} N\right|_{\mathbf{P}_{x}}\right) / T^{\prime} \mathbf{P}_{x}\right]=c_{1}\left(\left.T^{\prime} N\right|_{\mathbf{P}_{x}}\right)-c_{1}\left(T^{\prime} \mathbf{P}_{x}\right)=4-2=2 .
$$

As the general rank-2 holomorphic vector-bundle on $\mathbf{P}_{1}$ is $\mathscr{H}^{\otimes k} \oplus \mathscr{H}^{\otimes 2}$ (Grothendieck [1957]), we have

$$
\nu \cong \mathscr{H}^{\otimes(1-j)} \oplus \mathscr{H}^{\otimes(1+j)}
$$

for some $j \in \mathbf{Z}$. Let $V \subset \Gamma\left(\mathbf{P}_{x}, \mathcal{O}(\nu)\right)$ be the set of sections with zeros. If we had $j \neq 0$, then $V$ would be a vector subspace of $\Gamma(\mathcal{O}(\nu))$. Yet the canonical projection $\mathbf{P}_{x} \times \mathbf{C} T_{x} M \rightarrow \nu$ induces an inclusion $\mathbf{C} T_{x} M \rightarrow \Gamma(\mathcal{O}(\nu))$ with the property that the section corresponding to $v \in \mathbf{C} T_{x} M$ has a zero iff $g(v, v)=0$. (This follows from the equation

$$
\left.\left(\pi_{*} Д\right)\right|_{[b(w)]}=\operatorname{span}(w),
$$

where $w$ satisfies $g(w, w)=0$.) Hence $V$ is not a vector subspace, forcing $j=0$.

Knowing this, we are now in a position to prove the

MaIN TheOREM. Let $(M,[g])$ be a smooth conformal Riemannian 3-manifold, and let $(N, Д)$ be its twistor $C R$ manifold. Then $(N, Д)$ is imbeddable iff $M$ admits a real-analytic atlas for which $[g]$ is represented by a real-analytic metric.

Proof. Suppose that $(N, Z)$ is imbeddable as a real hypersurface in a complex 3-manifold $\mathscr{T}$. Let $\hat{\mathfrak{M}}$ denote the space of all $\mathbf{P}_{1}$ 's holomorphically imbedded in $\mathscr{T}$ with holomorphic normal bundle $\left(\left.T^{\prime} \mathscr{T}\right|_{\mathbf{P}_{1}}\right) / T^{\prime} \mathbf{P}_{1}$ isomorphic to $\mathscr{H} \oplus \mathscr{H}$.

Since $\mathbf{P}_{1}$ is a rigid manifold and $\mathscr{H} \oplus \mathscr{H}$ is a rigid bundle

$$
\left[H^{1}\left(\mathcal{O}\left((\mathscr{H} \oplus \mathscr{H}) \otimes(\mathscr{H} \oplus \mathscr{H})^{*}\right)\right)=\mathbf{C}^{4} \otimes H^{1}\left(\mathbf{P}_{1}, \mathcal{O}\right)=0\right],
$$

$\hat{\mathfrak{M}}$ is open in the space of all holomorphic compact curves in $\mathscr{T}$. Since

$$
H^{1}(\mathcal{O}(\mathscr{H} \oplus \mathscr{H}))=0,
$$

it follows (Kodaira [1962]) that $\hat{\mathfrak{M}}$ is a complex manifold with tangent space at $\mathscr{C} \in \hat{\mathfrak{M}}$ given by

$$
\Gamma(\mathscr{C}, \mathcal{O}(\nu)) \cong \Gamma\left(\mathbf{P}_{1}, \mathcal{O}(\mathscr{H} \oplus \mathscr{H})\right) \cong \mathbf{C}^{4},
$$

where $\nu$ is the holomorphic normal bundle of $\mathscr{C} \subset \mathscr{T}$; the manifold topology is, moreover, just the compact-open topology on $\hat{\mathfrak{M}}$ (Douady [1966]). The strategy of the proof is to now construct a complexification of $M$ as a complex hypersurface $\mathfrak{M}$ in the complex 4-manifold $\hat{\mathfrak{M}}$ and then show that $[g]$ has an analytic continuation to $\mathfrak{M}$.

Recall that $(N, I)$ comes equipped with a CR contact form $\theta \in \Omega^{1}(L)$ respected by an anti-CR involution $\rho: N \rightarrow N$ commuting with the projection $N \rightarrow M$. Since $L^{\otimes 2} \cong \Lambda^{3} T^{\prime} N=\left.\left(\Lambda^{3} T^{\prime} \mathscr{T}\right)\right|_{N}, L$ is a locally trivial CR line bundle, and extends as a holomorphic line-bundle $\hat{L}$ on some neighborhood of $N$ in $\mathscr{T}$ because $N$ is anticlastic. In the same way, the $L$-valued 1-form $\theta$ then extends as a holomorphic $\hat{L}$-valued contact form $\hat{\theta}$ on some neighborhood of $N$. Similarly, $\rho$ extends to a fixed-point free antiholomorphic involution $\hat{\rho}$ of some neighborhood of $N$. Let us call the common domain of these objects $\mathscr{T}_{0} \subset \mathscr{T}$, and let $\hat{\mathfrak{M}}_{0} \subset \hat{\mathfrak{M}}$ be the space of those curves $\mathscr{C} \in \hat{\mathfrak{M}}$ lying in $\mathscr{T}_{0}$. 
If $\mathscr{F}$ denotes the complex 5 -manifold given by

$$
\mathscr{F}:=\left\{(y, \mathscr{C}) \in \mathscr{T}_{0} \times \hat{\mathfrak{M}}_{0} \mid y \in \mathscr{C}\right\},
$$

and if $\alpha: \mathscr{F} \rightarrow \mathscr{T}_{0}$ and $\beta: \mathscr{F} \rightarrow \hat{\mathfrak{M}}_{0}$ are the canonical projections, we may define a holomorphic line-bundle $\kappa \rightarrow \mathscr{F}$ by setting $\kappa^{*}:=\operatorname{ker}\left[\beta_{*}: T^{\prime} \mathscr{F} \rightarrow T^{\prime} \hat{\mathfrak{W}}_{0}\right]$. The restriction of $\kappa$ to every fiber of $\beta$ is the cotangent bundle of the fiber and has Chern number -2 . On the other hand, the restriction of $\alpha^{*} \hat{L}$ to any fiber of $\beta$ has Chern number +2 by our previous calculation of the Chern number of $L$ on any fiber of $\pi$. Hence $\kappa \otimes \alpha^{*} \hat{L}$ is trivial on the fibers of $\beta$, and we may define a line-bundle $E \rightarrow \hat{\mathfrak{M}}_{0}$ by

$$
\mathcal{O}(E):=\beta_{*}^{0}\left(\mathcal{O}\left(\kappa \otimes \alpha^{*} \hat{L}\right)\right)
$$

i.e.

$$
E_{x}:=\Gamma\left(\beta^{-1}(x), \mathcal{O}\left(\kappa \otimes \alpha^{*} \hat{L}\right)\right)
$$

for all $x \in \hat{\mathfrak{M}}_{0}$. But $\alpha^{*} \hat{\theta} \in \Gamma\left(Q, \mathcal{O}\left(\alpha^{*} \hat{L} \otimes T^{*} Q\right)\right)$ defines a canonical section of $\kappa \otimes \alpha^{*} \hat{L}$ by restriction to the fibers of $\beta$, and thereby defines a canonical holomorphic section $f$ of $E$. Let $\mathfrak{M} \subset \hat{\mathfrak{M}}_{0}$ be the zero locus of $f$.

Suppose that $\mathscr{C} \in \mathfrak{M}$. Then $\hat{\theta}$ is orthogonal to $T^{\prime} \mathscr{C}$ and hence defines a section

$$
\check{\theta} \in \Gamma\left(\mathscr{C}, \mathcal{O}\left(\nu^{*} \otimes \hat{L}\right)\right) \cong \Gamma\left(\mathbf{P}_{1}, \mathcal{O}(1)+\mathcal{O}(1)\right) .
$$

Because $\hat{\theta}$, and hence $\check{\theta}$, has no zeros, contraction with $\check{\theta}$ yields an exact sequence

$$
0 \rightarrow \mathbf{C}_{\mathscr{C}} \rightarrow \underset{J_{\hat{\theta}}}{\rightarrow} \hat{L} \rightarrow 0
$$

of holomorphic vector bundles on $\mathscr{C}$, where $\mathbf{C}_{\mathscr{C}}$ denotes the trivial line-bundle. (The kernel of $\nu \rightarrow \hat{L}$ must be trivial because $c_{1}(\nu)=c_{1}(\hat{L})=2$.) Note that since $\left.H^{1}(\mathscr{C}, \mathcal{O})=0,\right\lrcorner_{\hat{\theta}}$ induces a surjection

$$
\Gamma(\mathscr{C}, \mathcal{O}(\nu)) \underset{\lrcorner_{\hat{\theta}}}{\rightarrow} \Gamma(\mathscr{C}, \mathcal{O}(\hat{L})) \cong \Gamma\left(\mathbf{P}_{1}, \mathcal{O}(2)\right) .
$$

We shall now show that $\mathfrak{M}$ is a complex 3-manifold with tangent space given by $\Gamma(\mathscr{C}, \mathcal{O}(\hat{L}))$ by showing that the defining function $f$ of $\mathfrak{M}$ has a nonzero derivative in the direction given by a section of $\mathbf{C}_{\mathscr{G}}$. Indeed, let $\gamma: \mathbf{P}_{1} \times B_{\boldsymbol{\varepsilon}} \rightarrow \mathscr{T}_{0}$ be a holomorphic map with $\gamma\left(\mathbf{P}_{1} \times\{0\}\right)=\mathscr{C}$ and

$$
\left.\left[\gamma_{*}(\partial / \partial z)\right]\right|_{t=0} \in \Gamma\left(\mathscr{C}, \mathcal{O}\left(\mathbf{C}_{\mathscr{C}}\right)\right)-\{0\}
$$

where $B_{\varepsilon}$ is a neighborhood of the origin in the complex $z$-plane $\mathbf{C}$; and let $w$ be a local nonzero holomorphic vector field on $\mathbf{P}_{1} \times B_{\varepsilon}$ tangent to the first factor and constant in the $z$-direction. Then, relative to any local trivialization of $\hat{L}$,

$$
\begin{aligned}
\frac{\partial}{\partial z}\left\langle\gamma^{*} \hat{\theta}, w\right\rangle & =\left(\gamma^{*} d \hat{\theta}\right)\left(\frac{\partial}{\partial z}, w\right)+w\left\langle\gamma^{*} \hat{\theta}, \frac{\partial}{\partial z}\right\rangle \\
& =\gamma^{*} d \hat{\theta}\left(\frac{\partial}{\partial z}, w\right),
\end{aligned}
$$


recalling that $\mathbf{C}_{\mathscr{C}}$ is the kernel of $\lrcorner_{\hat{\theta}}: \nu \rightarrow \hat{L}$. But since $\hat{\theta}$ is a contact form, $d \hat{\theta}$ is nondegenerate on the orthogonal space of $\hat{\theta}$, which is spanned by $\gamma_{*}(\partial / \partial z)$ and $\gamma_{*}(w)$ at $z=0$. Hence

$$
\left.\frac{\partial}{\partial z}\left\langle\gamma^{*} \hat{\theta}, w\right\rangle\right|_{z=0} \neq 0
$$

showing that the derivative at $z=0$ of $f$ along the curve $z \mapsto \gamma\left[\mathbf{P}_{1} \times\{z\}\right] \in \hat{\mathfrak{M}}_{0}$ is nonzero. Hence $d f \neq 0$ when $f=0$, and $\mathfrak{M}$ is a complex 3-manifold. Moreover, the projection $\lrcorner_{\hat{\theta}}: \quad \Gamma(\mathscr{C}, \mathcal{O}(\nu)) \rightarrow \Gamma(\mathscr{C}, \mathcal{O}(\hat{L}))$ takes $T_{\mathscr{C}}^{\prime} \mathfrak{M}$ isomorphically onto $\Gamma(\mathscr{C}, \mathcal{O}(\hat{L}))$.

We may now define a holomorphic conformal structure on $\mathfrak{M}$ via the above identification. By such a structure we mean a holomorphic line-bundle $E \subset$

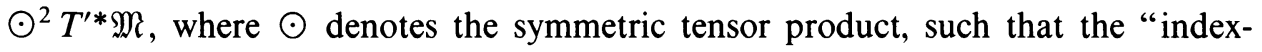
lowering map"

$$
\begin{aligned}
b_{E} & : T^{\prime} \mathfrak{M} \rightarrow T^{*} \mathfrak{M} \otimes E^{*} \\
\quad & : v \mapsto v\lrcorner \mathscr{I}
\end{aligned}
$$

is an isomorphism, where $\mathscr{I}$ is the holomorphic section of $\left(\odot^{2} T^{* * \mathfrak{M})} \otimes E^{*}\right.$ corresponding to the section 1 of $E \otimes E^{*}$. Associated with such a structure is a null-quadric distribution $Q \subset \mathbf{P}\left(T^{\prime} \mathfrak{M}\right)$, given by

$$
Q:=\left\{[v] \in \mathbf{P}\left(T^{\prime} \mathfrak{M}\right) \mid\langle b(v), v\rangle=0 \in E^{*}\right\} .
$$

The intersection of $Q$ with every fiber of $\mathbf{P}\left(T^{\prime} \mathfrak{M}\right) \rightarrow \mathfrak{M}$ is a nondegenerate quadric -i.e. a conic curve for $\operatorname{dim} \mathfrak{M}=3$, the case of present interest. Conversely, any submanifold of $\mathbf{P}\left(T^{\prime} \mathfrak{M}\right)$ intersecting every fiber in a nondegenerate quadric arises from exactly one holomorphic conformal structure (LeBrun [1983]).

Let $\hat{\beta}: \mathscr{F} \rightarrow \mathfrak{M}$ denote the restriction of the $\mathbf{P}_{1}$-bundle $\beta: \mathscr{F} \rightarrow \hat{\mathfrak{M}}_{0}$ to $\mathfrak{M} \subset \hat{\mathfrak{M}}_{0}$, and define a holomorphic vector bundle $T \rightarrow \mathfrak{M}$ by

$$
\mathcal{O}(T):=\hat{\beta}_{*}^{0}\left(\mathcal{O}\left(\alpha^{*} \hat{L}\right)\right),
$$

so that $T_{\mathscr{C}}=\Gamma(\mathscr{C}, \mathcal{O}(\hat{L}))$ for all $\mathscr{C} \in \mathfrak{M}$. Then $\hat{\theta}$ identifies $T$ with $T^{\prime} \mathfrak{M}$ in a holomorphic fashion by the previously considered map (contraction with normal bundles). Since $T_{x}=\Gamma\left(\hat{\beta}^{-1}(x), \mathcal{O}\left(\alpha^{*} \hat{L}\right)\right) \cong \Gamma\left(\mathbf{P}_{1}, \mathcal{O}(2)\right) \forall x \in \mathfrak{M}$, there is a canonical map

$$
\begin{aligned}
\mathscr{J}: \tilde{\mathscr{F}} & \rightarrow \mathbf{P}(T) \\
: y & \mapsto \Gamma\left(\hat{\beta}^{-1} \hat{\beta}(y), \widetilde{\Im}_{y}^{2} \otimes \mathcal{O}\left(\alpha^{*} \hat{L}\right)\right),
\end{aligned}
$$

where $\widetilde{\Im}_{y} \subset \mathcal{O}_{\hat{\beta}^{-1} \beta(y)}$ is the ideal of $y$; thus $\mathscr{J}$ has as its image those sections (up to scale) with their two zeros coinciding. Since the image of $\mathscr{J}$ meets every fiber of $\mathbf{P}(T) \rightarrow \mathfrak{M}$ in a conic, and since $T$ has been identified with $T^{\prime} \mathfrak{M}$, this defines a holomorphic conformal structure on $\mathfrak{M}$.

We now bring $\hat{\rho}$ into play. This antiholomorphic involution on $\mathscr{T}_{0}$ will take $\mathbf{P}_{1}$ 's with normal bundle_ $\mathscr{H} \oplus \mathscr{H}$ antiholomorphically to $\mathbf{P}_{1}$ 's with the same normal bundle. Since $\hat{\rho}^{*} \hat{\boldsymbol{\theta}}=\overline{\hat{\theta}}, \mathbf{P}_{1}$ 's orthogonal to $\hat{\boldsymbol{\theta}}$ are taken to $\mathbf{P}_{1}$ 's orthogonal to $\hat{\boldsymbol{\theta}}$. Hence $\hat{\rho}$ induces an antiholomorphic involution $\tilde{\rho}: \mathfrak{M} \rightarrow \mathfrak{M} ; \tilde{\rho}_{*} T^{\prime} \mathfrak{M}=\overline{T^{\prime} \mathfrak{M}}, \rho^{2}=1_{\mathfrak{M}}$. 
Since $\hat{\rho}$ respects $\hat{\theta}$, the null-quadric distribution defined by $\mathscr{J}$ will be taken to its complex conjugate by $\tilde{\rho}$.

Let $\hat{\mathfrak{M}}$ be the fixed point set $\tilde{\rho}$. Then $\hat{M}$ is a real-analytic 3-manifold with real-analytic conformal structure $[\hat{g}]$ induced by $E$; the space of complex directions for this real-analytic conformal structure is just the restriction of the null-quadric distribution to the "real slice" $\hat{M}$. But there is a canonical conformal isometry

$$
\iota: M \rightarrow \hat{M}, \quad \iota^{*}[\hat{g}]=[g]
$$

given by $x \mapsto \mathbf{P}_{x}, x \in M$ ! Indeed, by the lemma $\mathbf{P}_{x}$ has the correct normal bundle type in $\mathscr{T}_{0}$, is a fixed point of $\tilde{\rho}$, and $\iota_{*}$ takes complex null vectors to complex null vectors because $\left\langle\theta, \pi^{*} \nu\right\rangle, v \in \mathbf{C} T_{x} M$, has a double zero iff $v$ satisfies $g(v, v)=0$.

Thus, the imbeddability of $(N, Д)$ implies that $(M,[g])$ is conformally isometric to some real-analytic $(\hat{M},[\hat{g}])$. Since, conversely, $(N, \not)$ will be real-analytic with respect to a suitable atlas if $(M,[g])$ is, and since real-analyticity is a sufficient condition for the imbeddability of a CR manifold, we are done.

The reader may note that when $(M,[g])$ is analytic, we have imbedded $M$ in a complex 4-manifold. This 4-manifold admits a real slice induced by $\hat{\rho}$ and comes equipped with a self-dual Einstein metric (with scalar curvature -1 ) singular at $M$. For more details, cf. LeBrun [1982].

Finally, one may note that the existence of an atlas making a given conformal metric $[g]$ analytic is a nonvacuous requirement-provided, of course, that, as in the present case, the dimension exceeds 2 . For, by the use of partitions of unity, we may construct metrics which are conformally flat in some regions but not conformally flat elsewhere; this cannot occur for analytic metrics because of the principal of analytic continuation as applied to the Schouten tensor. This gives us a large class of nonimbeddable anticlastic CR 5-manifolds.

\section{REFERENCES}

M. Atiyah, N. Hitchin, and I. M. Singer [1978], Proc. Roy. Soc. London Ser. A 362, 425-464.

S. Bochner [1943], Ann. of Math. (2) 44, 652-673.

A. Douady [1966], Ann. Inst. Fourier (Grenoble) 16, 1-95.

A. Grothendieck [1957], Amer. J. Math. 79, 121-138.

K. Kodaira [1962], Ann. of Math. (2) 75, 146-162.

C. R. LeBrun [1982], Proc. Roy. Soc. London Ser. A 380, 171-185. [1983], Trans. Amer. Math. Soc. 278, 209-231.

H. Lewy [1956], Ann. of Math. (2) 64, 514-522.

L. Nirenberg [1973], Lectures on Linear Partial Differential Equations, Amer. Math. Soc., Providence, R. I.

R. Penrose [1975], Quantum Gravity (C. Isham et al., eds.), pp. 268-407. [1976], Gen. Relativity Gravitation 7, 31-52. [1983], Bull. Amer. Math. Soc. (N. S.) 8, 427-448.

A. Weinstein [1977], Lectures on Symplectic Manifolds, Amer. Math. Soc., Providence, R. I.

R. O. Wells, Jr. [1966], Proc. Amer. Math. Soc. 17, 1272-1275.

Department of Mathematics, State University of New York, Stony Brook, New York 11794 